COMUNICACIÓN CORTA

\title{
Determinación de la eficacia de antihelmínticos para el tratamiento de la gastroenteritis verminosa en caprinos de la provincia de Tucumán
}

\author{
Chocobar $\mathrm{MLE}^{1}$, Diambra $\mathrm{P}^{2}$, Terán Alurralde $\mathrm{MR}^{2}$, Bustos $\mathrm{MJ}^{2}$, Prado $\mathrm{JM}^{2}$, \\ Gonzalez Moreno $\mathrm{C}^{2,3}$, Fernández $\mathrm{JL}^{2}$, Salinas $\mathrm{C}^{2}$, Solaligue $\mathrm{PBV}^{2}$, Rosa $\mathrm{RJ}^{1,2^{*}}$ \\ ${ }^{1}$ Faculdade de Medicina Veterinária e Zootecnia - Câmpus de Botucatu, Universidade Estadual Paulista, \\ São Paulo, Brasil. \\ ${ }^{2}$ Facultad de Agronomía y Zootecnia, Universidad Nacional de Tucumán, Argentina. \\ 3 Instituto Superior de Investigaciones Biológicas (Consejo Nacional de Investigaciones Científicas y \\ Técnicas - Universidad Nacional del Litoral), Tucumán, Argentina. \\ * Correspondencia: Ramiro José Rosa, Cátedra de Clínica de Grandes Animales - Facultad de \\ Agronomía y Zootecnia - UNT. Finca "El Manantial". Florentino Ameghino s/n., Bo Mercantil, CP \\ 4105, El Manantial, Tucumán, Argentina. E-mail: ramirorosa19@hotmail.com
}

Recibido: 8 Agosto 2020. Aceptado: 7 Octubre 2020. Disponible en línea: 4 Noviembre 2020

Editor: P. Beldomenico

RESUMEN. En la Argentina, el 93\% de la producción caprina en la Argentina es desarrollada por pequeños productores de agriculturas de subsistencia, quienes, por lo general, utilizan antihelmínticos sin asesoramiento profesional, lo que, probablemente, ha llevado al desarrollo de resistencia, comprometiendo seriamente la sustentabilidad del control de nematodos. El objetivo de este estudio es determinar la eficacia de la ivermectina, el albendazol y el levamisol para el tratamiento de la gastroenteritis verminosa en cuatro grupos de ocho caprinos cada uno, mediante el Test de Reducción del Conteo de Huevos (TRCH). Los resultados demostraron un $100 \%$ de reducción del número de HPG para los grupos tratados con albendazol y levamisol y un $17 \%$ de reducción para el grupo tratado con ivermectina, con respecto al grupo control, lo que permite inferir que existe resistencia a este fármaco.

SUMMARY. Determination of the efficacy of anthelmintics used in the control of gastrointestinal nematodes of goats from Tucumán, Argentina. Almost $93 \%$ of goat production in Argentina is developed by small farmers, who generally use anthelmintics without professional advice, which has led to the development of resistance, seriously compromising the sustainability of nematode control. This study aims to determine the efficacy of ivermectin, albendazole and levamisole for the treatment of verminous gastroenteritis in four groups of eight goats each, using the Faecal Egg Count Reduction Test (FECRT). The results showed a 100\% reduction in the number of EPG for the groups treated with albendazole and levamisole and a $17 \%$ reduction for the group treated with ivermectin, compared to the control group, which allows us to infer that there is resistance to this drug.

Palabras clave: caprinos, gastroenteritis verminosa, resistencia a antihelmínticos, ivermectina

Keywords: goats, verminous gastroenteritis, anthelmintic resistance, ivermectin

\section{Introducción}

La producción caprina en la Argentina cuenta, actualmente, con 4,7 millones de cabezas distribuidas en las regiones más áridas del país, concentrando en el NOA un $34 \%$ de los animales (Chagra Dib et al., 2018). Se estima que el $93 \%$ de la producción caprina está en manos de pequeños productores e íntimamente ligada a economías de subsistencia que utilizan a la cría extensiva sobre pastizales naturales como un medio para afianzarse en el territorio y ejercer la soberanía (Alvarez et al., 2003; SENASA, 2015).

Los parásitos gastrointestinales de los caprinos producen efectos negativos sobre la producción, provocan- do disminución de la ganancia de peso de las cabras madres, afectando los parámetros reproductivos y la producción de $\mathrm{kg}$ de carne de cabritos mamones, y pudiendo producir hasta la muerte de animales jóvenes (Rossanigo, 2007). La necesidad de controlar las nematodiosis determina la aplicación de tratamientos frecuentes y masivos sobre el hato completo, por lo general sin diagnostico previo o control profesional, favoreciendo la aparicion de resistencia a las drogas (Caracostantogolo et al., 2012).

Existe una proporción de parásitos que poseen Resistencia Antihelmíntica (RA) debido a múltiples causas. Las principales se centran en la alta frecuencia de desparasitaciones, el uso indiscriminado de antipara- 
sitarios y la falta de rotación de principios activos (Fiel et al., 2001), además del concepto de refugio, que en parasitología se define como la proporción de la población parasitaria que no es expuesta a la acción del control, siendo, para el caso de los nemátodos gastrointestinales, la población de parásitos en estadios de vida libre que se encuentra en el pasto (Buffarini, 2011). La implementación de programas de control parasitario que consideren el concepto de refugio es fundamental para mantener una población parasitaria en la pastura que no esté sujeta a la selección por tratamientos antihelmínticos, reduciendo así la aparición de RA (Suarez y Cristel, 2014, Suarez et al., 2014).

En general, los fármacos antihelmínticos en caprinos son utilizados a partir de las dosis recomendadas para ovinos y bovinos. Sin embargo, se ha demostrado que, en la especie caprina, la absorción de los antihelmínticos es menor que en otros rumiantes y que, a la vez, los metabolizan y eliminan de forma más rápida (Anziani y Fiel, 2015; Cooper et al., 2016).

El sistema FAMACHA, basado en la evaluacion de la mucosa conjuntival como indicador del nivel de anemia causada por los nematodos (sobre todo Haemonchus contortus), es una valiosa herramienta de ayuda diagnóstica a campo, ya que permite aplicar tratamientos quimicos selectivos a los caprinos más afectados por parásitos hematófagos, dejando sin tratar al resto del hato, a fin de reducir el surgimiento de RA (Aguirre y Cafrune, 2013). El uso de dicho sistema para caprinos lecheros del Noroeste argentino fue validado por Suarez et al (2014); el cual reportó que la sensibilidad de la técnica fue del $100 \%$ cuando se consideraron positivos los valores de FAMACHA de 3 a 5, aunque la especificidad fue del $13,2 \%$. Sin embargo, cuando sólo fueron considerados positivos los valores de 4 y 5 , la especificidad aumentó a 84,9\%, datos importantes a considerar para evitar aplicar tratamiento a animales negativos, y, de esta forma, evitar los fenómenos de RA. Asimismo, la RA está comprometiendo seriamente la sustentabilidad del control de nematodos, lo que resulta en importantes pérdidas económicas y, a su vez, ha llevado a la revalorización del conocimiento y entendimiento de los procesos que se interrelacionan en la tríada animal-medioambiente-parásito, con el objetivo de idear programas de Control Parasitario Sustentable (CPS); siendo responsabilidad de la Medicina Veterinaria replantear el enfoque tradicional de los programas de control parasitario (Fleming, 2010; Fiel et al., 2011; Anziani y Fiel, 2015; Cooper et al., 2016).

Es por los motivos expuestos que el presente estudio tiene como objetivo determinar la eficacia de los antihelmínticos ivermectina, albendazol y levamisol para el tratamiento de la GEV en caprinos a campo, mediante el uso del TRCH.

\section{Materiales y Métodos}

El estudio se desarrolló en el Centro Experimental Caprino (CEC) de la Facultad de Agronomía y Zootecnia de la Universidad Nacional de Tucumán (Tucumán, Argentina), ubicado en El Manantial, a $8 \mathrm{~km}$ al Sureste de la ciudad de San Miguel de Tucumán, a 26951' de latitud Sur y 65017' de Longitud Oeste y a una altitud de 440 m.s.n.m. La precipitación media anual es de 1.100 $\mathrm{mm}$ y las lluvias se producen principalmente entre los meses de octubre y marzo. La temperatura media anual es de 19,4우 C; la del mes más cálido (enero) es de 24,90 C y la del mes más frío (julio) de 12,1ํ C. El clima es de tipo subtropical - sub - húmedo con estación seca.

Se utilizaron 32 caprinos mayores de 18 meses, de raza mestiza (Criolla x Anglo Nubian), sin distinción de sexo, pertenecientes al CEC. Los animales en estudio solían ser desparasitados cada 3 meses, utilizando Ivermectina $1 \%$ en dosis de $1 \mathrm{ml}$ cada $50 \mathrm{~kg}$ de peso vivo, administrada a todo el hato, y habían recibido tratamiento por última vez en el mes de septiembre de 2019.

Los animales fueron pesados y posteriormente distribuidos de manera aleatoria para conformar 4 grupos de 8 animales cada uno: Control sin Tratamiento (C); Tratamiento con Albendazol (TA); Tratamiento con Ivermectina (TI) y Tratamiento con Levamisol (TL).

La toma y remisión de muestras fue realizada en dos momentos, ambos en el mes de noviembre del año 2019. En el momento 1 (M1) se tomaron de 20 a $30 \mathrm{gr}$ de muestras de materia fecal por masaje rectal de cada animal, siguiendo la técnica descrita por Fiel et al (2011) $y$, posteriormente, se aplicó el tratamiento definido para cada grupo, como puede observarse en la Tabla 1.

Tabla 1. Distribución de grupos y tratamientos con antihelmínticos, así como la dosis y vía de administración.

$\mathrm{N} / \mathrm{A}=$ No Aplicable; $\mathrm{C}=$ Grupo Control; $\mathrm{TA}=$ Tratamiento con Albendazol; $\mathrm{Tl}=$ Tratamiento con Ivermectina; $\mathrm{TL}=$ Tratamiento con Levamisol.

\begin{tabular}{|l|l|l|l|}
\hline Grupos & Droga & Dosis & $\begin{array}{l}\text { Vía de } \\
\text { administración }\end{array}$ \\
\hline $\mathbf{C}(\mathbf{n}=\mathbf{8})$ & N/A & N/A & N/A \\
\hline TA $(\mathbf{n = 8})$ & Albendazol & $7,5 \mathrm{mg} / \mathrm{kg}$ & Oral \\
\hline TI $(\mathbf{n}=\mathbf{8})$ & Ivermectina & $400 \mu \mathrm{mg} / \mathrm{kg}$ & Subcutánea \\
\hline TL $(\mathbf{n}=\mathbf{8})$ & Levamisol & $8 \mathrm{mg} / \mathrm{kg}$ & Subcutánea \\
\hline
\end{tabular}

Para la administración de ivermectina y levamisol fueron utilizadas 16 jeringas descartables estériles de $5 \mathrm{ml}$ con aguja descartable de tamaño $25 \times 8$. El alben-dazol fue administrado por vía oral con el uso de 8 jeringas descartables estériles.

Las muestras fueron remitidas dentro de bolsas de nylon de $10 \times 20 \mathrm{~cm}$, cerradas, sin aire en su interior y rotuladas con el número de caravana de cada animal, además de la sigla del grupo al que pertenecen. Las mismas fueron transportadas en cajas de telgopor refrigeradas con hielo hasta el Laboratorio de la 
Facultad de Agronomía y Zootecnia, Finca el Manantial donde fueron almacenadas a $4^{\circ} \mathrm{C}$ hasta su procesamiento al día siguiente.

La extracción de materia fecal se repitió en el momento 2 (M2), 14 días después del M1. En este momento no fue administrado ningún antihelmíntico y la remisión, transporte y procesamiento de las muestras fueron idénticos a los realizados en el M1. La eficacia de las drogas utilizadas fue estimada a través de la comparación del recuento de HPG presente en el M1 y M2 para cada grupo utilizando la técnica de McMaster Modificada por Robert y O'Sullivan (1949), descrita por Fiel et al. (2011) y aplicando, poste-riormente, el TRCH en materia fecal. Para ello, se calculó la media aritmética de los recuentos de HPG en el M1 y M2 utilizando el programa InfoStat ${ }^{\circledR}$.

Seguidamente se aplicó la Ecuación 1, descrita por Dash et al (1988), que correlaciona la media de HPG de cada grupo tratado en el $\mathrm{M} 1$ y $\mathrm{M} 2$ respecto al grupo control en ambos momentos, permitiendo obtener el porcentaje de Reducción de HPG tras el tratamiento instaurado para cada grupo.

Ecuación 1: Cálculo de Reducción de HPG. La fórmula correlaciona la media aritmética de HPG de los grupos tratados en el $\mathrm{M} 1$ y $\mathrm{M} 2$ con respecto a la media aritmética de HPG del grupo control sin tratamiento (Dash et al 1988).

\section{$\mathrm{TRCH}=100 \times(1-[\mathrm{T} 2 / \mathrm{T} 1][\mathrm{C} 1 / \mathrm{C} 2])$}

Donde $\mathrm{T} 2$ = media aritmética de HPG del grupo tratado en el $\mathrm{M} 2, \mathrm{~T} 1$ = media aritmética de HPG para el grupo tratado en el M1, C1 = media aritmética de HPG del grupo control en el M1, $\mathrm{C} 2$ = media aritmética de HPG del grupo control sin tratamiento en el $\mathrm{M} 2$

El análisis de varianza no paramétrico (Kruskal Wallis), con nivel de significancia del 95\%, fue utilizado como herramienta principal en el estudio estadístico del efecto de los tratamientos antiparasitarios. Un valor de $\mathrm{P} \leq 0,05$ se consideró estadísticamente significativo. El procesamiento de los datos fue realizado mediante la utilización del software R (The R Project for Statistical Computing).

\section{Resultados}

La Tabla 2 reúne los valores de HPG que presentaban los animales en estudio en el M1 y M2. Las medias aritméticas y los desvíos estándar de los recuentos de HPG para cada grupo tratado y del grupo control en el M1 y M2 se encuentran en la Tabla 3.

El recuento de HPG por la técnica de McMáster modificada mostró una reducción post-tratamiento (M2) en los 4 grupos ensayados. Sin embargo, los resultados obtenidos tras la aplicación de la Ecuación 1 indican que el TRCH luego de los tratamientos (M2) fue de $100 \%$ para los grupos TA y TL mientras que para el grupo TI fue de $17 \%$ (Figura 1) para este grupo de animales bajo las condiciones de estudio aplicadas.

Para el análisis estadístico los resultados se presentaron como valores medios y rangos intercuartiles (IQ). EI análisis de la variancia no paramétrico (Kruskal Wallis) al $5 \%$ de la diferencia del recuento de HPG entre M1 y M2 mostró que existen diferencias significativas entre los grupos $(p=0,01235)$, sin embargo, el análisis de prueba de los rangos con signo de Wilcoxon evidenció que solo se observan diferencias significativas entre TA con respecto a $\mathrm{TI}(p=0,027)$ para este grupo de animales bajo las condiciones de estudio aplicadas (Tabla 4).

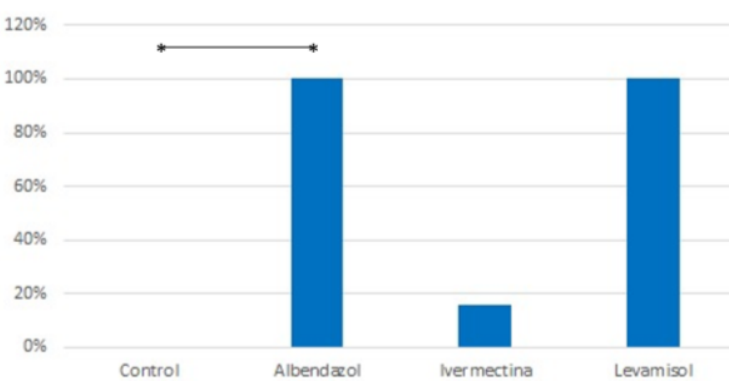

Figura 1. Test de Reducción del Conteo de Huevos (TRCH) en los grupos tratados con antihelmínticos. Control: Sin tratamiento, TA: Tratamiento con Albendazol; TI: Tratamiento con Ivermectina; TL: Tratamiento con Levamisol. * $(p<0,05)$.

\section{Discusión}

En el M1 la mayoría de los individuos analizados presentaba recuentos de HPG superiores al límite de 500 HPG descrito por Aguirre et al (2002), lo que indica la presencia de nematodos responsables de la GEV en gran parte de los animales estudiados. Estos datos pueden explicarse teniendo en cuenta que dichas cabras fueron criadas en confinamiento y se alimentaron de pasturas naturales lo cual coincide con lo expuesto por Caracostantogolo et al. (2012) y Cardillo (2012). Ellos reportaron que el uso de pastizales naturales para la alimentación animal y la intervención del ser humano en la domesticación de los animales son prácticas que tienden a aumentar el riesgo de transmisión de parasitosis. Sin embargo, una importante limitante del presente estudio fue la falta de identificación de especies parasitarias presentes en el hato, tema que será objetivo de estudios posteriores

Los resultados evidenciaron una reducción de los valores de HPG para el grupo control en el M2, sobre el cual no fue aplicado tratamiento. Esta reducción en el número de HPG puede deberse a características propias del animal, producto de la interacción del sistema inmune del individuo con el parásito, como afirma Caracostantogolo et al. (2012), considerando, además, que dicha resistencia inmunológica a las parasitosis es capaz de disminuir o anular totalmente la oviposición en gran parte de los géneros parasitarios (Fiel et al., 2011). Es por este motivo que la recomendación profesional para la desparasitación de hatos caprinos 
debe basarse en la observación de signos clínicos de parasitosis, sumado a la utilización del método FAMACHA, y no administrar antihelmínticos a aquellos que son naturalmente resistentes a los nematodes.

Tabla 2. Recuento de HPG en M1 y M2.

\begin{tabular}{|c|c|c|c|}
\hline $\begin{array}{l}\text { Número de } \\
\text { Muestra }\end{array}$ & Caravana & HPG M1 & HPG M2 \\
\hline \multicolumn{4}{|c|}{ GRUPO CONTROL (C) } \\
\hline 1 & 45 & 600 & 40 \\
\hline 2 & 713 & 1400 & 0 \\
\hline 3 & 721 & 320 & 220 \\
\hline 4 & 92 & 940 & 660 \\
\hline 5 & 194 & 500 & 240 \\
\hline 6 & 195 & 320 & 1820 \\
\hline 7 & 197 & 1220 & 340 \\
\hline 8 & 53 & 640 & 160 \\
\hline \multicolumn{4}{|c|}{ TRATAMIENTO CON ALBENDAZOL (TA) } \\
\hline 9 & 22 & 940 & 0 \\
\hline 10 & 705 & 1400 & 0 \\
\hline 11 & 708 & 600 & 0 \\
\hline 12 & 725 & 1160 & 0 \\
\hline 13 & 34 & 1240 & 0 \\
\hline 14 & 49 & 600 & 0 \\
\hline 15 & 57 & 720 & 0 \\
\hline 16 & 60 & 920 & 0 \\
\hline \multicolumn{4}{|c|}{ TRATAMIENTO CON IVERMECTINA (TI) } \\
\hline 17 & T13 & 940 & 20 \\
\hline 18 & 018/003 & 300 & 440 \\
\hline 19 & 712 & 540 & 0 \\
\hline 20 & $36 / 30$ & 380 & 220 \\
\hline 21 & 99/001 & 300 & 60 \\
\hline 22 & $51 / 23$ & 1120 & 1400 \\
\hline 23 & 56 & 940 & 380 \\
\hline 24 & 59 & 600 & 0 \\
\hline \multicolumn{4}{|c|}{ TRATAMIENTO CON LEVAMISOL (TL) } \\
\hline 25 & 2 & 1400 & 0 \\
\hline 26 & $50 / 010$ & 1040 & 0 \\
\hline 27 & 701 & 720 & 0 \\
\hline 28 & 702 & 540 & 0 \\
\hline 29 & 714 & 940 & 0 \\
\hline 30 & 719 & 600 & 0 \\
\hline 31 & $91 / 12$ & 380 & 0 \\
\hline 32 & 200 & 520 & 0 \\
\hline
\end{tabular}

Teniendo en cuenta que la ivermectina redujo los recuentos de HPG en un $17 \%$ respecto al grupo control bajo las condiciones de estudio aplicadas $y$, considerando que cualquier valor de TRCH por debajo del $90 \%$ se asocia a la presencia de RA (Fiel et al., 2001), es posible inferir que existe resistencia a la droga utilizada. Caracostantogolo et al. (2012) y Anziani y Fiel (2015) afirman que la ivermectina es el antihelmíntico más comúnmente utilizado en hatos y que, por lo general, el fármaco es administrado sin supervisión profesional, situación que puede contribuir a la aparición de dicha resistencia en el hato estudiado. Los animales de este estudio eran rutinariamente desparasitados mediante la administración de ivermectina $1 \%$, utilizando la dosis recomendada para bovinos $(1 \mathrm{ml}$ cada $50 \mathrm{~kg}$ de peso vivo) sin considerar la presencia de signos clínicos ni aplicar la técnica de FAMACHA. Esta situación puede explicar la baja eficacia de esta droga para el control de las parasitosis en el hato.

Tabla 3. Media aritmética y desvío estándar de HPG en M1 y M2. $\mathrm{C}=$ Grupo Control $\mathrm{TA}=$ Tratamiento con Albendazol; $\mathrm{Tl}=$ Tratamiento con Ivermectina; $\mathrm{TL}=$ Tratamiento con Levamisol.

\begin{tabular}{|c|c|c|c|c|}
\hline Grupo & $\begin{array}{c}\text { Media } \\
\text { de HPG en } \\
\text { M1 }\end{array}$ & $\begin{array}{c}\text { Desv. } \\
\text { estándar } \\
\text { M1 }\end{array}$ & $\begin{array}{c}\text { Media } \\
\text { de HPG en } \\
\text { M2 }\end{array}$ & $\begin{array}{c}\text { Desv. } \\
\text { estándar } \\
\text { M2 }\end{array}$ \\
\hline $\begin{array}{c}\text { C } \\
(\mathbf{n}=\mathbf{8})\end{array}$ & $\mathbf{7 4 2 ~ H P G}$ & 404,6074 & 435 HPG & 595,58 \\
\hline $\begin{array}{c}\text { TA } \\
(\mathbf{n}=\mathbf{8})\end{array}$ & 947 HPG & 299,8928 & 0 HPG & 0,0 \\
\hline $\begin{array}{c}\text { TI } \\
(\mathbf{n}=\mathbf{8})\end{array}$ & 640 HPG & 320,8916 & 315 HPG & 471,56 \\
\hline $\begin{array}{c}\text { TL } \\
(\mathbf{n}=8)\end{array}$ & 767 HPG & 337,5436 & 0 HPG & 0,0 \\
\hline
\end{tabular}

Tabla 4. Comparaciones por pares utilizando la prueba de suma de rangos de Wilcoxon (valor $p$ ). $\mathrm{C}=$ Grupo Control TA= Tratamiento con Albendazol; $\mathrm{Tl}=$ Tratamiento con Ivermectina; $\mathrm{TL}=$ Tratamiento con Levamisol. ${ }^{* *}$ Estadísticamente significativo $(p<0,05)$.

\begin{tabular}{|c|c|c|c|}
\hline & $\mathbf{C}$ & TA & TI \\
\hline TA & 0,136 & - & - \\
\hline TI & 0,834 & $0,027^{* *}$ & - \\
\hline TL & 0,345 & 0,49 & 0,263 \\
\hline
\end{tabular}

Por otro lado, si bien solo se observaron diferencias significativas en la reducción de recuentos de HPG entre el grupo control y aquel tratado con albendazol, la reducción en los grupos tratados con albendazol y levamisol fue de $100 \%$, con lo cual queda demostrada la eficacia de dichos fármacos para el tratamiento de la GEV en los animales estudiados. Este resultado es de gran importancia ya que, actualmente, las únicas presentaciones comerciales de antihelmínticos para uso exclusivo en pequeños rumiantes aprobadas por SENASA son las que contienen estos grupos químicos 
(imidazotiazoles y benzimidazoles) (Anziani y Fiel, 2015).

Teniendo en cuenta los resultados obtenidos podemos concluir que los agentes causales de GEV en el hato estudiado presentan resistencia al tratamiento con ivermectina, pero son sensibles a tratamientos con levamisol y albendazol en las dosis recomendadas para la especie caprina.

El estudio y entendimiento de las interacciones que ocurren dentro de la tríada hospedador-ambienteparásito y la detección de fenómenos de resistencia a antihelmínticos en hatos contribuyen al desarrollo de alternativas de Control Parasitario Sustentable, favoreciendo no solamente la salud animal sino también la salud humana y las actividades económicas relacionadas a la producción caprina.

La identificación de los géneros parasitarios resistentes mediante el uso de la técnica de cultivo y recuperación de larvas infectivas $L 3$, así como la determinación de la infectividad de las pasturas exceden los objetivos de este trabajo, sin embargo, serán técnicas consideradas en estudios futuros.

\section{Agradecimientos}

Al Decano de la Facultad de Agronomía y Zootecnia, Prof. Mag. Ing. Agr. Roberto D. Corbella, por cedernos parte del Laboratorio de Edafología para el procesamiento de las muestras, parte fundamental del desarrollo de este estudio. Al Prof. Vet. Domingo Viscido por el apoyo brindado durante todo el desarrollo de este trabajo además de la participación en la lectura y corrección de los esbozos de este manuscrito.

\section{Bibliografía}

Aguirre DH, Cafrune MM, Viñabal AE, Salatin AO. 2002. Aspectos epidemiológicos y terapéuticos de la nematodiasis gastrointestinal caprina en un área subtropical de la Argentina. RIA 31: 114-123.

Alvarez JD, Racioppi O, Recalde G, Viola Resconi J, Moriena RA. 2003. Eficacia de la ivermectina para controlar parásitos gastrointestinales en caprinos. Rev. Vet. UNNE 14: 2.

Anziani OS, Fiel CA. 2015. Resistencia a los antihelmínticos en nematodos que parasitan a los rumiantes en la argentina. RIA 41: 34-46.

Buffarini M. 2011. Resistencia a los antiparasitarios. ¿Cómo diagnosticarla? Memoria Técnica INTA. https://inta.gob.ar/sites/default/files/script-tmpmt2011 buffarini resistencia antiparasitarios como.pdf

Caracostantogolo J, Schapiro J, Morici G, Castaño Zubieta R, Martinez M. 2012. Enfermedades parasitarias en rumiantes: gastroenteritis verminosa. En: Rosa $A$, Ribicich $M$.
Parasitología y Enfermedades Parasitarias en Veterinaria. Hemisferio Sur, Buenos Aires. Pp. 135-143.

Cardillo N. 2012. Generalidades de Parasitología. En: Rosa A, Ribicich M. Parasitología y Enfermedades Parasitarias en Veterinaria. Ed. Hemisferio Sur, Buenos Aires. Pp. 23-27.

Cooper L, Cerutti J, Mihn C, Torrents J, Suarez Archilla G, Anziani OS. 2016. Eficacia del monepantel para el control de aislamientos de Haemonchus contortus y Trichostrongylus spp. con resistencia múltiple (ivermectina y febendazole) en caprinos. FAVE Sección Ciencias Veterinarias 15: 5-8.

Dash K, Hall K, Barger IA. 1988. The role of arithmetic and geometric worm egg counts in faecal eggcount reduction test and in monitoring strategic drenching programs in sheep. Austr. Vet. J. 65: 66-68.

Fiel CA, Steffan PE, Ferreyra DA. 2011. Diagnóstico de las parasitosis más frecuentes de los rumiantes: técnicas de diagnóstico e interpretación de resultados. Material de divulgación inédito, Tandil.

Fiel CA, Anziani OS, Suarez V, Vazquez R, Eddi C, Romero J, Caracostantógolo J, Saumell C, Mejía M, Costa J, Steffan P. 2001. Resistencia antihelmíntica en bovinos, causas, diagnóstico y profilaxis. Rev. Vet. Arg. 171: 21-32.

Fleming S. 2010. Programas de Control Parasitario. En: Smith B. Medicina Interna de Grandes Animales. Ed. Elsevier, Barcelona. Pp. 1623-1647.

Rossanigo CE. 2007. Parasitosis de las cabras. En: Suarez VH, Olaechea FV, Rossanigo CE y Romero JR. Enfermedades parasitarias de los ovinos y otros rumiantes menores en el cono sur de América. INTA. San Luis. Pp: 247-270.

Suarez VH y Cristel SL. 2014. Risk factors for anthelmintic resistance development in cattle gastrointestinal nematodes in Argentina. Rev. Bras. de Parasitol. Vet. 23: 129-135.

Suarez VH, Frondraz M, Viñabal AE y Salatin AO. 2014. Validación del método FAMACHA para detectar anemia en caprinos lecheros en los valles templados del Noroeste argentino. Rev. Med. Vet. 95: 4-11.

SENASA. 2015. Cadena Animal: Caprinos. Recuperado el 20 de 06 de 2020, de http://www.senasa.gob.ar/cadenaanimal/caprinos 